\title{
Boundary contributions of on-shell recursion relations with multiple-line deformation
}

\author{
Chang $\mathrm{Hu}^{\mathrm{a}}$, Xiao-Di $\mathrm{Li}^{\mathrm{b}}{ }^{\mathrm{D}}$, Yi Li ${ }^{\mathrm{c}}$ \\ Department of Physics, Zhejiang Institute of Modern Physics, Zhejiang University, No. 38 Zheda Road, Hangzhou 310027, People's Republic \\ of China
}

Received: 3 September 2020 / Accepted: 3 October 2020 / Published online: 19 October 2020

(C) The Author(s) 2020

\begin{abstract}
The on-shell recursion relation has been recognized as a powerful tool for calculating tree-level amplitudes in quantum field theory, but it does not work well when the residue of the deformed amplitude $\hat{A}(z)$ does not vanish at infinity of $z$. However, in such a situation, we still can get the right amplitude by computing the boundary contribution explicitly. In Arkani-Hamed and Kaplan (JHEP 04:076. https://doi.org/10.1088/1126-6708/2008/04/ 076. arXiv:0801.2385, 2008), the background field method was first used to analyze the boundary behaviors of amplitudes with two deformed external lines in different theories. The same method has been generalized to calculate the explicit boundary operators of some amplitudes with BCFWlike deformation in Jin and Feng (JHEP 04:123. https://doi. org/10.1007/JHEP04(2016)123. arXiv:1507.00463, 2016). In this paper, we will take a step further to generalize the method to the case of multiple-line deformation, and to show how the boundary behaviors (even the boundary contributions) can be extracted in the method.
\end{abstract}

\section{Contents}

1 Introduction ................ 1

2 Boundary term of on-shell recursion relation . . . . 2

3 Real scalar field theory . . . . . . . . . . . . . . 3

4 Yukawa theory . . . . . . . . . . . . . 5 5

5 Yang-Mills theory . . . . . . . . . . . . 9

6 Conclusion . . . . . . . . . . . . . . 12

References . . . . . . . . . . . . . . . . . . 12

\footnotetext{
a e-mail: liyiphysics@zju.edu.cn

b e-mail: lixiaodi@zju.edu.cn (corresponding author)

c e-mail: alberthu@zju.edu.cn
}

\section{Introduction}

Recent decades have witnessed the prosperity in the area of scattering amplitudes, including the discovery of new mathematical structures, the new formalisms of scattering amplitudes, and even more important, the new methods for calculating the scattering amplitude more efficiently. Among these methods the on-shell recursion relations, pioneered by the BCFW recursion relations $[3,4]$, have been proved to be very powerful tools, which can be used to construct higher-point tree-level amplitudes from lower-point ones. After the idea of deforming two external momenta to capture the analytic structures of tree-level amplitudes was introduced, quickly the deformation of multiple lines and even all lines was used in [5-7] to discuss on-shell constructibility or other aspects of the amplitudes.

The on-shell recursion relations are based on the Cauchy theorem. It says that under an appropriate deformation of a subset of $n$ momenta, $p_{i} \rightarrow \hat{p}_{i}(z)=p_{i}+z q_{i}$, with $z$ being a complex parameter, the residue of $\hat{A}_{n}(z) / z$ at $z=0$, which is nothing but the $n$-point physical amplitude $A_{n}$, equals the minus of the sum of all other residues. We divide the latter into two parts: $A_{n}=-\sum_{z_{I}} \operatorname{Res}_{z=z_{I}} \hat{A}_{n}(z) / z-B_{n}$. Here $\operatorname{Res}_{z=z_{I}} \hat{A}_{n}(z) / z$ is a residue at finite $z_{I}$ and it factorizes into $\sum_{z_{I}} \hat{A}_{L}\left(z_{I}\right) \frac{1}{P_{I}^{2}} \hat{A}_{R}\left(z_{I}\right)$ with $\hat{A}_{L}$ and $\hat{A}_{R}$ being lower-point amplitudes, while $B_{n}$ is the residue at $z=\infty$ and does not have a similar factorization, called the boundary contribution (or boundary term). Then we can see that if the boundary contribution vanishes, the on-shell recursion relations provide an efficient method for calculating $n$-point amplitudes by lower-point amplitudes, but these relations will meet with problems when $B_{n}$ does not vanish, which means $\hat{A}_{n}(z)$ does not vanish at $z=\infty$. $^{1}$

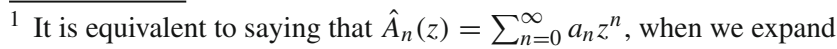
it around infinity.
} 
Since the boundary term $B_{n}$ is vital for the on-shell recursion relation, many methods were proposed to deal with it. The first step is to determine for which theories the boundary contributions vanish in the on-shell recursion relations. In $[1,8]$, the authors demonstrated that the deformed amplitudes of a wide variety of theories vanish at infinity by splitting the scattering process into a hard part and a soft background. However, there are also some theories or some cases where the boundary contributions do not vanish. Then in $[9,10]$, the authors choose to introduce auxiliary fields to eliminate the boundary term. However, if the boundary term does exist, we can also try to separate $B_{n}$ from others and compute it explicitly. Then in [11-13] the authors try to isolate the boundary term by analyzing the properties of Feynman diagrams. And in [14], collecting factorization limits of all physical poles is applied to finding the boundary contribution. Another progress in this direction was made in [15-17], where the idea of expressing boundary terms as roots of amplitudes are introduced, although it is not very practical. Then in $[2,18-$ 20], multiple steps of a BCFW-like deformation were used to calculate the boundary contribution step by step until getting the final results.

However, all these methods can only be applied to limited types of theories, and thus a more general method is needed. Hence, we want to develop a general method which is applicable for broader theories. In $[1,7,8]$, the background field method was proved to be a good method to analyze the boundary behavior of deformed amplitudes; then in [2] the authors used the background field method to calculate the boundary term explicitly in the case of two momenta being deformed. All these suggest that we can generalize this background field method to more general cases of multiple-leg deformation for better efficiency. Although the same idea has been exploited in $[1,7,8]$, and especially in [7] the boundary behavior of deformed amplitudes of a lot of different theories in four dimensions was analyzed, we should emphasize that our objective is to compute the boundary term explicitly rather than just analyze the boundary behavior, and our discussions are in a general number of dimensions.

Now we roughly explain the basic idea. First when $z \rightarrow$ $\infty, m$ deformed momenta $\hat{p}_{i}(z) \sim z q_{i}$ are much larger than the other undeformed momenta $p_{j}$, so the original scattering process can be regarded as the process of some hard particles, $\hat{p}_{i}(z)$, scattering in the soft background of other soft particles, $p_{j}$. From this point of view, the $z$-dependence of $m$-deformed $n$-point amplitude $\widetilde{B}_{m}(z)^{2}$ is nothing but the $m$-point scattering amplitudes in the nontrivial soft background, which can be calculated by the corresponding Feynman diagrams. With such an understanding, the technical difficulty becomes the

2 The $z$-dependent part of the $m$-deformed $n$-point amplitude $\widetilde{B}_{m}(z)$ is not exactly equal to the boundary term $B_{n}$, since $\lim _{z \rightarrow \infty} \hat{A}_{n}(z) \approx$ $\widetilde{B}_{m}(z)$, and $B_{n}=\operatorname{Res}_{z=\infty} \hat{A}_{n}(z) / z \approx \operatorname{Res}_{z=\infty} \widetilde{B}_{m}(z) / z$. reading of the Feynman rules, including the interactive vertices and the nontrivial propagators, which will be discussed carefully in this paper.

The structure of the paper is as follows. In Sect. 2, we briefly introduce the method of the background field to calculate the boundary term. In Sect. 3, we begin to consider the simplest example of real scalar theory. Then in Sect. 4, the boundary contribution in Yukawa theory is computed and some results of examples are represented. In Sect. 5, we give the general discussions of Yang-Mills theory, then apply it to some explicit examples. In Sect. 6, we give the conclusion.

\section{Boundary term of on-shell recursion relation}

In this paper we will follow the method proposed in [2], which will be briefly reviewed in this section. In [2], the authors considered an $n$-point correlation function with two deformed momenta given by ${ }^{3}$

$$
\begin{aligned}
\hat{G}_{n}^{(2)}(z) & =\left\langle\Phi\left(\hat{p}_{1}(z)\right) \Phi\left(p_{2}\right) \cdots \Phi\left(\hat{p}_{n}(z)\right)\right\rangle \\
& =\int D \Phi e^{i S[\Phi]} \Phi\left(\hat{p}_{1}(z)\right) \Phi\left(p_{2}\right) \cdots \Phi\left(\hat{p}_{n}(z)\right) .
\end{aligned}
$$

After splitting the field $\Phi$ into a high energy (or hard) part $\Phi^{\Lambda}$ and a soft part (again denoted by $\Phi$ ) according to the energy scale $\Lambda \sim\left|z q_{i}\right| \gg p_{j}$, and expanding the action, then the leading contribution is

$\hat{G}_{n}^{(2)}(z)=\int D \Phi e^{i S[\Phi]} \hat{G}_{2}(z) \Phi_{2}\left(p_{2}\right) \cdots \Phi_{n-1}\left(p_{n-1}\right)$,

with

$\hat{G}_{2}(z)=\int \mathcal{D} \Phi^{\Lambda} \exp \left(i S_{2}^{\Lambda}\left[\Phi^{\Lambda}, \Phi\right]\right) \Phi_{1}^{\Lambda} \Phi_{n}^{\Lambda}$,

where $S_{2}^{\Lambda}\left[\Phi^{\Lambda}, \Phi\right]$ is the sum of terms quadratic in $\Phi^{\Lambda}$ in the expansion of the action. ${ }^{4}$ After applying the LSZ reduction to the $n$-point correlation function with two hard fields, we can get the large $z$-dependent part of the deformed amplitude

\footnotetext{
${ }^{3}$ Here we consider the $n$-point correlation function in momentum space. Under the LSZ reduction, each field $\Phi$ in $x_{i}$ of the correlation function $\left\langle\Phi\left(x_{1}\right) \Phi\left(x_{2}\right) \cdots \Phi\left(x_{n}\right)\right\rangle$ in position space is associated with an external momentum $p_{i}$ in amplitude. So if we make the twoline deformation $p_{1} \rightarrow \hat{p}_{1}(z), p_{n} \rightarrow \hat{p}_{n}(z)$ for an amplitude $A_{n}$, then the corresponding fields $\Phi\left(x_{1}\right), \Phi\left(x_{n}\right)$ in the correlation function are assigned $\hat{p}_{1}$ and $\hat{p}_{n}$ by LSZ reduction.

4 The terms linear in $\Phi^{\Lambda}$ vanish because of the equation of motion, and in the case of two hard particles we only need to consider quadratic terms, ignoring higher terms.
} 
with only two legs being deformed,

$$
\begin{aligned}
& (2 \pi)^{4} \delta^{4}\left(\sum_{i} \hat{p}_{i}\right) i \hat{A}_{n}(z) \\
& \quad=\left(i \lim _{\hat{p}_{1}^{2} \rightarrow 0} \hat{p}_{1}^{2}\right)\left(i \lim _{\hat{p}_{n}^{2} \rightarrow 0} \hat{p}_{n}^{2}\right) \hat{G}_{2} \prod_{j=2}^{N-1}\left(i \lim _{p_{j}^{2} \rightarrow 0} p_{j}^{2}\right) G_{n-2} .
\end{aligned}
$$

Since only $\hat{G}_{2}$ depends on $z$, the question of calculating the $z$-dependence of a deformed amplitude $\widetilde{B}_{n}(z)$ is transformed to the calculation of the two-point correlation function of hard fields $\hat{G}_{2}(z)$ in a soft background.

The above consideration is limited to the case with only two external momenta deformed, and only the terms quadratic in $\Phi^{\Lambda}$ contribute. We want to generalize this method to the multi-leg deformed case, for example, the Risagger deformation in [5]. Similar to the two-leg deformed case, the central part is the corresponding $m$-leg deformed correlation function

$$
\hat{G}_{m}(z)=\int \mathcal{D} \Phi^{\Lambda} \exp \left\{i S^{\Lambda}\left[\Phi^{\Lambda}, \Phi\right]\right\} \Phi_{1}^{\Lambda} \Phi_{2}^{\Lambda} \cdots \Phi_{m}^{\Lambda},
$$

where $S^{\Lambda}\left[\Phi^{\Lambda}, \Phi\right]$ is the hard part of the expansion of the action after splitting the field. After doing the LSZ reduction, it will become the large $z$-dependent part of a $m$-leg deformed amplitude $\widetilde{B}_{m}(z)$, just like (2.4). Particularly, this large $z$-dependent part $\hat{G}_{m}(z)$ can be calculated by Feynman diagrams of hard fields in the soft background.

Now we show how to read off Feynman rules in the nontrivial soft background. After splitting the fields into a hard part and a soft part, we expand the Lagrangian,

$$
\begin{aligned}
\mathcal{L}\left(\tilde{\Phi}, \partial_{\mu} \tilde{\Phi}\right) & =\mathcal{L}\left(\Phi+\Phi^{\Lambda}, \partial_{\mu} \Phi+\partial_{\mu} \Phi^{\Lambda}\right) \\
& =\mathcal{L}\left(\Phi, \partial_{\mu} \Phi\right)+\sum_{i} \frac{\partial \mathcal{L}}{\partial \tilde{\Phi}_{i}} \Phi_{i}^{\Lambda} \\
& +\sum_{i} \frac{\partial \mathcal{L}}{\partial\left(\partial_{\mu} \tilde{\Phi}_{i}\right)} \partial_{\mu} \Phi_{i}^{\Lambda} \\
& +\frac{1}{2 !} \sum_{i, j} \frac{\partial^{2} \mathcal{L}}{\partial \tilde{\Phi}_{i} \partial \tilde{\Phi}_{j}} \Phi_{i}^{\Lambda} \Phi_{j}^{\Lambda} \\
& +\frac{1}{2 !} \sum_{i, j} \frac{\partial^{2} \Phi}{\partial\left(\partial_{\mu} \tilde{\Phi}_{i}\right) \partial\left(\partial_{\nu} \tilde{\Phi}_{j}\right)} \partial_{\mu} \Phi_{i}^{\Lambda} \partial_{\nu} \Phi_{j}^{\Lambda} \\
& +\sum_{i, j} \frac{\partial^{2} \Phi}{\partial \tilde{\Phi}_{i} \partial\left(\partial_{\mu} \tilde{\Phi}_{j}\right)} \Phi_{i}^{\Lambda} \partial_{\mu} \Phi_{j}^{\Lambda}+\cdots \\
& =\mathcal{L}\left(\Phi, \partial_{\mu} \Phi\right) \\
& +\frac{1}{2 !} \sum_{i, j} \frac{\partial^{2} \mathcal{L}}{\partial \tilde{\Phi}_{i} \partial \tilde{\Phi}_{j}} \Phi_{i}^{\Lambda} \Phi_{j}^{\Lambda}
\end{aligned}
$$

$$
\begin{aligned}
& +\frac{1}{2 !} \sum_{i, j} \frac{\partial^{2} \Phi}{\partial\left(\partial_{\mu} \tilde{\Phi}_{i}\right) \partial\left(\partial_{\nu} \tilde{\Phi}_{j}\right)} \partial_{\mu} \Phi_{i}^{\Lambda} \partial_{\nu} \Phi_{j}^{\Lambda} \\
& +\sum_{i, j} \frac{\partial^{2} \Phi}{\partial \tilde{\Phi}_{i} \partial\left(\partial_{\mu} \tilde{\Phi}_{j}\right)} \Phi_{i}^{\Lambda} \partial_{\mu} \Phi_{j}^{\Lambda} \\
& +\frac{1}{3 !} \sum_{i, j, k} \frac{\partial^{3} \mathcal{L}}{\partial \tilde{\Phi}_{i} \partial \tilde{\Phi}_{j} \partial \tilde{\Phi}_{k}} \Phi_{i}^{\Lambda} \Phi_{j}^{\Lambda} \Phi_{k}^{\Lambda} \\
& +\frac{1}{2 !} \sum_{i, j, k} \frac{\partial^{3} \mathcal{L}}{\partial \tilde{\Phi}_{i} \partial \tilde{\Phi}_{j} \partial\left(\partial_{\mu} \tilde{\Phi}_{k}\right)} \Phi_{i}^{\Lambda} \Phi_{j}^{\Lambda} \partial_{\mu} \Phi_{k}^{\Lambda}+\cdots,
\end{aligned}
$$

where $\cdots$ represents higher order terms of $\Phi_{i}^{\Lambda}$ and we have used the integration by part and the equation of motion in the third equation to eliminate the terms linear in $\Phi_{i}^{\Lambda}$. From the above expansion, we can easily see that we do not need to consider $\mathcal{L}\left(\Phi, \partial_{\mu} \Phi\right)$, which is not involved with the hard fields $\Phi_{i}^{\Lambda}$.

There exist terms that are quadratic of $\Phi_{i}^{\Lambda}$, which will produce the nontrivial propagators by their inverse, just like in the ordinary Lagrangian. One non-trivial thing is that in general all fields $\Phi_{i}^{\Lambda}$ are mixed together through the coefficients of quadratic terms, then physically these coefficients act as different propagators linking with different fields (or a particle of one type changes into another type in the process of propagation). And the derivatives in those coefficients are important for the $z$-dependence of the deformed amplitude, since the derivatives produce deformed momenta in the Feynman rules.

There are also higher order terms of $\Phi_{i}^{\Lambda}$, which produce interaction vertices. When we consider $m$-leg deformed amplitudes, correspondingly we should consider $m$-leg Feynman diagrams of hard fields in the soft background. This means that if we want to compute the $m$-deformed correlation function $\hat{G}_{m}(z)$, we should consider the Feynman diagrams with $m$ hard external lines constructed by an $k$-point vertex with $k \leq m$. For example, in the 2-deformed case, we only consider $S_{2}^{\Lambda}\left[\Phi^{\Lambda}, \Phi\right]$, which only gives a propagator with two hard external lines, and in the 3-deformed case, we should consider the terms in the expansion of action up to cubic order of $\Phi_{i}^{\Lambda}$ and the corresponding Feynman diagram is constructed by three-vertices. In the rest of paper, we will mainly focus on three-leg deformed case to demonstrate our main idea, and there are no differences for the more general $m$-leg deformed case.

\section{Real scalar field theory}

In this section, we will focus on the simplest theory, i.e., real scalar field theory, as an example to show our method. More complicated theories will be considered in later sec- 


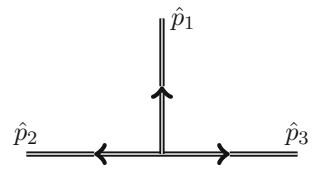

Fig. 1 The Feynman digram in soft background contributing for $\left\langle\Phi_{1} \Phi_{2} \Phi_{3}\right\rangle$. Here double solid lines represent the propagators of hard particles in a soft background

tions. From now on, we are limited to the three-leg deformed case. The Lagrangian of real scalar field theory we are considering is

$\mathcal{L}=-\frac{1}{2} \partial_{\mu} \phi \partial^{\mu} \phi+\frac{\lambda_{m}}{m !} \phi^{m}$

with $m \geq 3$. We make the substitution $\phi \rightarrow \phi+\Phi$ with $\Phi$ representing the hard part of the field and $\phi$ representing the soft part; then the expansion is

$$
\begin{aligned}
\mathcal{L}= & -\frac{1}{2} \partial_{\mu}(\phi+\Phi) \partial^{\mu}(\phi+\Phi)+\frac{\lambda_{m}}{m !}(\phi+\Phi)^{m} \\
= & -\frac{1}{2} \partial_{\mu} \phi \partial^{\mu} \phi+\frac{\lambda_{m}}{m !} \phi^{m} \\
& -\frac{1}{2} \partial_{\mu} \Phi \partial^{\mu} \Phi+\frac{\lambda_{m}}{2 !(m-2) !} \phi^{m-2} \Phi^{2}+\cdots+\frac{\lambda_{m}}{m !} \Phi^{m} \\
= & \mathcal{L}(\phi)+\mathcal{L}(\phi, \Phi) .
\end{aligned}
$$

Here we have omitted the terms proportional to $\Phi$ following the same arguments as in the previous section. $\mathcal{L}(\phi)$ represents the soft part of the Lagrangian, and $\mathcal{L}(\phi, \Phi)$ represents the hard part of the Lagrangian, called the hard Lagrangian.

We regroup $\mathcal{L}(\phi, \Phi)$ into a quadratic part and a higher order part, then the quadratic terms of $\Phi$ produce the propagator and the other terms give the interactive vertices

$$
\begin{aligned}
\mathcal{L}(\phi, \Phi)= & \frac{1}{2} \Phi\left(\partial^{2}+\frac{\lambda_{m}}{(m-2) !} \phi^{m-2}\right) \Phi \\
& +\frac{\lambda_{m}}{3 !(m-3) !} \phi^{m-3} \Phi^{3}+\cdots+\frac{\lambda_{m}}{m !} \Phi^{m} \\
= & \frac{1}{2} \Phi D \Phi+\mathcal{L}_{1}(\phi, \Phi) .
\end{aligned}
$$

Here we have used integration by parts and ignored the total derivative. We can read off the Feynman rules for propagator and vertices from the hard Lagrangian directly: the propagator is $-i D^{-1}$ with $\partial^{2}$ replaced by $-p^{2}$ and the three-vertex is $i \frac{\lambda_{m}}{(m-3) !} \phi^{m-3}$. Because we only consider a three-leg deformation, then only the cubic vertex can contribute. For the deformed correlation function, $\left\langle\Phi_{1} \Phi_{2} \Phi_{3}\right\rangle$, there is only one contributing Feynman diagram with a cubic vertex and three hard propagator lines, as shown in Fig. 1.

Now we calculate explicitly the $z$-dependent part of a $n$ point amplitude of real scalar theory. After doing the LSZ reduction, the deformed correlation function $\hat{G}_{3}(z)$ becomes $\widetilde{B}_{3}(z)$

$$
\begin{aligned}
i \widetilde{B}_{3}(z)= & \left(i \lim _{\hat{p}_{1}^{2} \rightarrow 0} \hat{p}_{1}^{2}\right)\left(i \lim _{\hat{p}_{2}^{2} \rightarrow 0} \hat{p}_{2}^{2}\right)\left(i \lim _{\hat{p}_{3}^{2} \rightarrow 0} \hat{p}_{3}^{2}\right) \hat{G}_{3}(z) \\
= & i \lim _{\hat{p}_{1}^{2} \rightarrow 0} \lim _{\hat{p}_{2}^{2} \rightarrow 0} \lim _{\hat{p}_{3}^{2} \rightarrow 0} \hat{p}_{1}^{2} \hat{p}_{2}^{2} \hat{p}_{3}^{2} D_{1}^{-1} D_{2}^{-1} D_{3}^{-1} \frac{\lambda_{m}}{(m-3) !} \phi^{m-3} \\
= & i \frac{1}{-1+\frac{1}{\hat{P}_{1}^{2}} \frac{\lambda_{m}}{(m-2) !} \phi^{m-2}} \frac{1}{-1+\frac{1}{\hat{P}_{2}^{2}} \frac{\lambda_{m}}{(m-2) !} \phi^{m-2}} \\
& \times \frac{1}{-1+\frac{1}{\hat{P}_{3}^{2}} \frac{\lambda_{m}}{(m-2) !} \phi^{m-2}} \frac{\lambda_{m}}{(m-3) !} \phi^{m-3} \\
= & -i \frac{\lambda_{m} \phi^{m-3}}{(m-3) !} \sum_{i=0}^{\infty} \sum_{j=0}^{\infty} \sum_{k=0}^{\infty}\left[\frac{\lambda_{m}}{(m-2) !} \phi^{m-2} \frac{1}{\hat{P}_{1}^{2}}\right]^{i} \\
& \times\left[\frac{\lambda_{m}}{(m-2) !} \phi^{m-2} \frac{1}{\hat{P}_{2}^{2}}\right]^{j}\left[\frac{\lambda_{m}}{(m-2) !} \phi^{m-2} \frac{1}{\hat{P}_{3}^{2}}\right]^{k} \\
= & -i \frac{\lambda_{m} \phi^{m-3}}{(m-3) !}\left[1+\frac{\lambda_{m}}{(m-2) !} \phi^{m-2} \frac{1}{\hat{P}_{1}^{2}}\right. \\
& \left.+\frac{\lambda_{m}}{(m-2) !} \phi^{m-2} \frac{1}{\hat{P}_{2}^{2}}+\frac{\lambda_{m}}{(m-2) !} \phi^{m-2} \frac{1}{\hat{P}_{3}^{2}}+\cdots\right]
\end{aligned}
$$

where we expanded the propagators and dots represent higher order terms in the expansion. Now we address the physical meaning of the above formula. In (3.4), each double-line propagator in soft background is expanded by a geometric series with each term being a product of a propagator $1 / \hat{P}^{2}$ and a vertex $\phi^{m-2}$ to some power, and the expansion can be depicted by the diagram in Fig. 2. In Diagram 2, every solid line represents a propagator of the hard field without soft background, every two-vertex represents a real vertex connected with $(m-2)$ soft lines and 2 hard lines and the cubic vertex connected with $(m-3)$ soft lines and 3 hard lines because of the interaction term $\frac{\lambda_{m}}{3 !(m-3) !} \phi^{m-3} \Phi^{3}$. Then we can see that the diagram in Fig. 1 exactly describes the propagation and interaction of hard particles in a soft background. So $\widetilde{B}_{3}(z)$ represents the complete contribution of all Feynman diagrams containing the boundary part of deformed amplitude $\hat{A}_{n}(z)$.

From the general picture, we can specify the boundary behavior or large $z$ behavior of deformed amplitude $\hat{A}_{n}(z)$. If we choose the 3 -deformed momenta $\hat{p}_{i}(z)=p_{i}+z q_{i}$ with $i=1,2,3$ satisfying the conditions

$q_{i} \cdot q_{j}=0$ with $i, j=1,2,3$;

$p_{i} \cdot q_{i}=0$, with $i=1,2,3$,

we will get $\hat{p}_{i}^{2}=0$, i.e., the on-shell condition for three hard legs. We should emphasize that although $\hat{p}_{i}^{2}=0$, the 
Fig. 2 The Feynman diagram represents the real scatting process without soft background after expanding the double-line propagators in Fig. 1

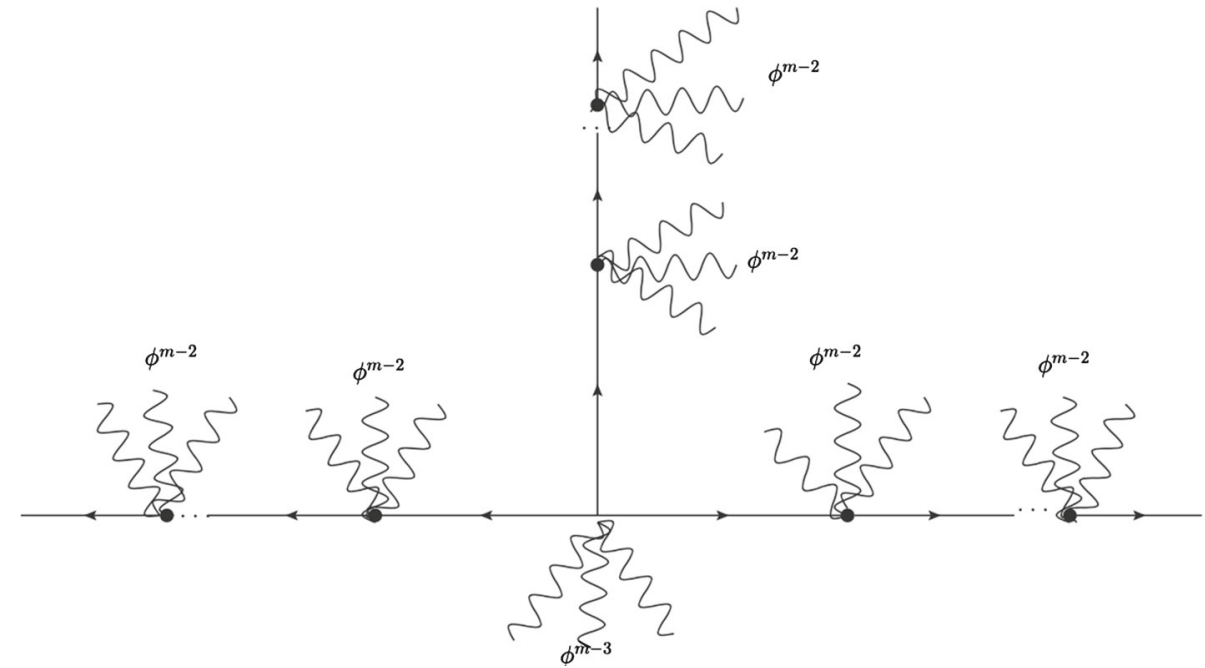

expansion in (3.4) is $\hat{P}_{i}$, which contains not only the hard momentum $\hat{p}_{i}$, but some soft momenta $p_{j}$ 's $(j=4, \ldots, n)$ for the vertex $\frac{\lambda_{m}}{(m-2) !} \phi^{m-2}$. With this explanation, we see that

$$
\begin{aligned}
\frac{1}{\left(\hat{p}_{i}+P_{S}\right)^{2}} & =\frac{1}{\left(p_{i}+z q_{i}+P_{S}\right)^{2}} \\
& =\frac{1}{2 P_{S} \cdot p_{i}+2 z P_{S} \cdot q_{i}+P_{S}^{2}} \sim \frac{1}{z},
\end{aligned}
$$

where $P_{S}=\sum_{j \in S} p_{j}$ with $S \subset\{4, \ldots, n\}$ and in the last step we take $z \rightarrow \infty$. Then the contribution of every propagator $1 / \hat{P}_{i}^{2}$ in (3.4) is $O\left(\frac{1}{z}\right)$, so we can easily see that the leading term of the expansion, $\frac{\lambda_{m}}{(m-3) !} \phi^{m-3}$, is of the order $O(1)$ and all other terms in the expansion vanish for the additional propagators $1 / \hat{P}_{i}^{2}$. We conclude that $\widetilde{B}_{3}(z)$ is non-vanishing in the three-leg deformed amplitude of real scalar theory.

Although the above method is general, however the threeleg deformed case is a little special since the diagram in Fig. 1 has only a cubic vertex and does not have any internal propagators. Then a question arises: what happens if we make a four-deformation or even higher $s$-deformation? Now let us assume we have made a $s$-leg deformation with $s \geq 4$. The situation becomes a little different for there are two cases, $m \geq s$ and $m<s$, where $m$ is the power of the leading term in (3.1). If $m \geq s$, then in the expansion of Lagrangian there is always a term $\phi^{m} \Phi^{m-s}$ which will give a Feynman diagram having only one $(m-s)$-leg vertex, and the boundary contribution of this diagram does not vanish, so in this case the boundary contribution does not vanish for the $s$-leg deformed on-shell recursion relation. For the second case $m<s$, all interaction terms in the expansion of Lagrangian are like $\phi^{m} \Phi^{m-t}$ with $t<s$, so all contributing diagrams for the $z$-dependent part of $s$-leg deformed amplitude must have an extra hard propagator which acts as $O\left(\frac{1}{z}\right)$; in this case the boundary contribution vanishes. So for the real scalar Lagrangian with finite terms, we can always use on-shell recursion relations with enough legs being deformed, whose boundary term vanishes. ${ }^{5}$

\section{Yukawa theory}

In this section, we will move on to consider a little more complicated theory, i.e. Yukawa theory. The Lagrangian of the Yukawa theory considered here is ${ }^{6}$

$\mathcal{L}=-\frac{1}{2} \partial^{\mu} \phi \partial_{\mu} \phi+i \bar{\psi} \gamma^{\mu} \partial_{\mu} \psi+\lambda \bar{\psi} \psi \phi$

In comparison with the real scalar field theory, Yukawa theory shows some differences. First, the appearance of fermionic fields $\psi$ and $\bar{\psi}$ bring about some extra minus signs in the process of calculation for commutating two fermionic fields. Second, since the number of fields is higher than 1 and they interact with each other, then after doing the expansion, there will appear some propagators connecting different fields. ${ }^{7}$ For these reasons, the number of diagrams we should consider will be higher than 1 .

Just like before, we split the fields into hard parts and soft parts: $\phi \rightarrow H+\phi, \bar{\psi} \rightarrow \bar{\Psi}+\psi, \psi \rightarrow \Psi+\psi$, then the Lagrangian is also divided into two parts:

$$
\begin{aligned}
\mathcal{L} & =\mathcal{L}(\phi, \psi, \bar{\psi})+\mathcal{L}(H, \bar{\Psi}, \Psi) \\
& =-\frac{1}{2} \partial^{\mu} \phi \partial_{\mu} \phi+i \bar{\psi} \gamma^{\mu} \partial_{\mu} \psi+\lambda \bar{\psi} \psi \phi
\end{aligned}
$$

\footnotetext{
${ }^{5}$ Using the terminology in other literature, the real scalar theory with finite interaction terms is always on-shell constructible.

${ }^{6}$ Here we do not consider the interaction of saclar field.

${ }^{7}$ It is because we are only considering the hard fields and ignoring the soft fields, that there appear such unphysical propagators. But if we do the series expansion of these propagators, then we will find they are actually physical, just like Fig. 2.
} 


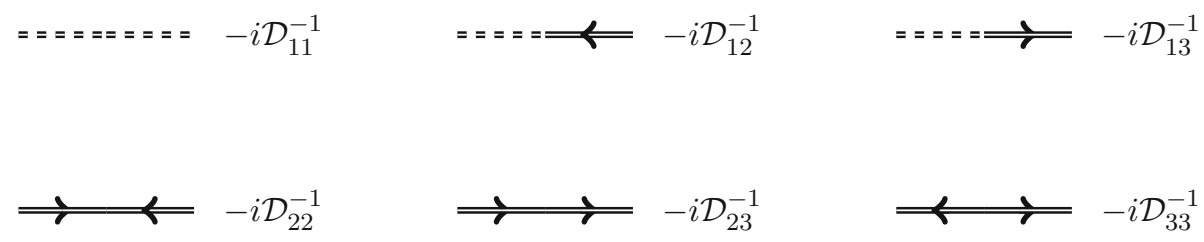

Fig. 3 The six propagators for hard fields in soft background. Here a dashed double-line represents a propagating scalar boson, while a solid double-line represents a propagating fermion, and the arrow represents the direction of the propagation of a fermion

$$
\begin{aligned}
& -\frac{1}{2} \partial^{\mu} H \partial_{\mu} H+i \bar{\Psi} \gamma^{\mu} \partial_{\mu} \Psi+\lambda \bar{\psi} \Psi H \\
& +\lambda \bar{\Psi} \psi H+\lambda \bar{\Psi} \Psi \phi+\lambda \bar{\Psi} \Psi H,
\end{aligned}
$$

where we have used the equations of motion to drop those terms proportional to hard fields. Then we only focus on the hard part of the Lagrangian and recast it in the form

$$
\begin{aligned}
\mathcal{L}(H, \bar{\Psi}, \Psi)= & -\frac{1}{2} \partial^{\mu} H \partial_{\mu} H+i \bar{\Psi} \gamma^{\mu} \partial_{\mu} \Psi \\
& +\lambda \bar{\psi} \Psi H+\lambda \bar{\Psi} \psi H+\lambda \bar{\Psi} \Psi \phi+\lambda \bar{\Psi} \Psi H \\
= & -\frac{1}{2}\left(H \bar{\Psi} \Psi^{T}\right) \\
& \times\left(\begin{array}{ccc}
-\partial^{2} & \lambda \psi^{T} & -\lambda \bar{\psi} \\
-\lambda \psi & 0 & -i \gamma^{\mu} \vec{\partial}_{\mu}-\lambda \phi \\
\lambda \bar{\psi}^{T} & i\left(\gamma^{\mu}\right)^{T} \overleftarrow{\partial}_{\mu}+\lambda \phi & 0
\end{array}\right) \\
& \times\left(\begin{array}{c}
H \\
\bar{\Psi}^{T} \\
\Psi
\end{array}\right)+\lambda \bar{\Psi} \Psi H \\
= & -\frac{1}{2}\left(H \bar{\Psi} \Psi^{T}\right) D\left(\begin{array}{c}
H \\
\bar{\Psi}^{T} \\
\Psi
\end{array}\right)+\lambda \bar{\Psi} \Psi H \\
= & \mathcal{L}_{0}(H, \Psi, \bar{\Psi})+\mathcal{L}_{1}(H, \Psi, \bar{\Psi}),
\end{aligned}
$$

where we have used integration by parts and transposition of fermionic fields like $\bar{\Psi} \Psi=-\Psi^{T} \bar{\Psi}^{T}, \bar{\Psi} \gamma^{\mu} \partial_{\mu} \Psi=$ $-\left(\partial_{\mu} \Psi^{T}\right)\left(\gamma^{\mu}\right)^{T} \bar{\Psi}^{T}$. Here $\mathcal{L}_{0}(H, \Psi, \bar{\Psi})$ represents the free part of hard Lagrangian, from which we can get the expressions of propagators, and $\mathcal{L}_{1}(H, \Psi, \bar{\Psi})$ is the interaction part, which gives use the cubic vertex. And we can simply infer that there are six propagators for $D$ being antisymmetric.
To get the concrete expressions for these propagators, we first divide the matrix $D$ into two parts, $D_{0}$ and $V$, because the inverse of $D_{0}$ is easy to calculate, then we apply the geometric series expansion to $D$,

$$
\begin{aligned}
D^{-1} & =\left(D_{0}+V\right)^{-1}=\left[D_{0}\left(1+D_{0}^{-1} V\right)\right]^{-1} \\
& =\sum_{k=0}^{\infty}(-1)^{k}\left(D_{0}^{-1} V\right)^{k} D_{0}^{-1} \\
& =D_{0}^{-1}-D_{0}^{-1} V D_{0}^{-1}+D_{0}^{-1} V D_{0}^{-1} V D_{0}^{-1}+\cdots,
\end{aligned}
$$

with

$D_{0}=\left(\begin{array}{ccc}-\partial^{2} & 0 & 0 \\ 0 & 0 & -i \overrightarrow{\not \partial} \\ 0 & i \check{\not \partial}^{T} & 0\end{array}\right), \quad V=\left(\begin{array}{ccc}0 & \lambda \psi^{T} & -\lambda \bar{\psi} \\ -\lambda \psi & 0 & -\lambda \phi \\ \lambda \bar{\psi}^{T} & \lambda \phi & 0\end{array}\right)$,

where we have used the formula of inverse of a multiplication of two operators, $(A B)^{-1}=B^{-1} A^{-1}$. The inverse of $D_{0}$ is easy to get as

$D_{0}^{-1}=\left(\begin{array}{ccc}-\frac{1}{\partial^{2}} & 0 & 0 \\ 0 & 0 & \frac{-i \overleftarrow{\not} T}{\partial^{2}} \\ 0 & \frac{i \vec{\partial}}{\partial^{2}} & 0\end{array}\right)$,

where the arrows represent the directions of the action of the derivatives in numerators, and we can easily check $D_{0} D_{0}^{-1}=$ $D_{0}^{-1} D_{0}=1 .^{8}$ So the inverse of $D$ is given by the expansion (4.4) as

\footnotetext{
${ }^{8}$ We choose the convention $\left\{\gamma^{\mu}, \gamma^{\nu}\right\}=2 g^{\mu \nu}$.
} 


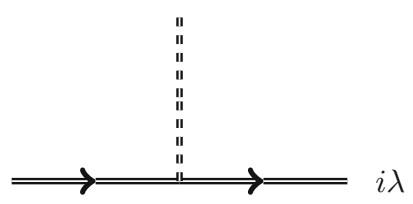

Fig. 4 Three-vertex in soft background for Yukawa theory, $i \lambda$ expansion of $D^{-1}$, new things appear:

$$
\begin{aligned}
\frac{\partial_{1}}{\partial_{1}^{2}} \frac{\partial_{2}}{\partial_{2}^{2}} & =\frac{\partial_{1} \cdot \partial_{2}}{\partial_{1}^{2} \partial_{2}^{2}} \sim \frac{\hat{p}_{1} \cdot \hat{p}_{2}}{\hat{p}_{1}^{2} \hat{p}_{2}^{2}} \\
& =\frac{p_{1} \cdot p_{2}+2 z\left(p_{1} \cdot q_{2}+p_{2} \cdot q_{1}\right)}{\left(p_{1}^{2}+2 z p_{1} \cdot q_{1}\right)\left(p_{2}^{2}+2 z p_{2} \cdot q_{2}\right)} \sim \frac{1}{z},
\end{aligned}
$$

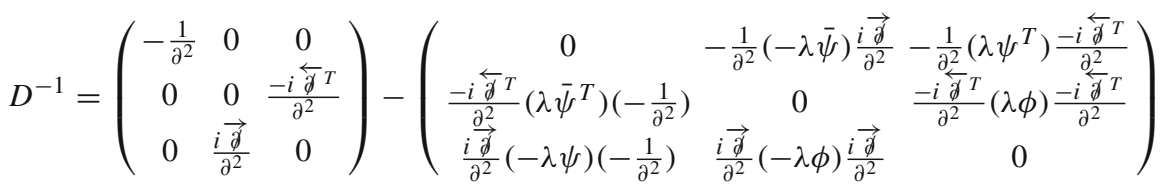

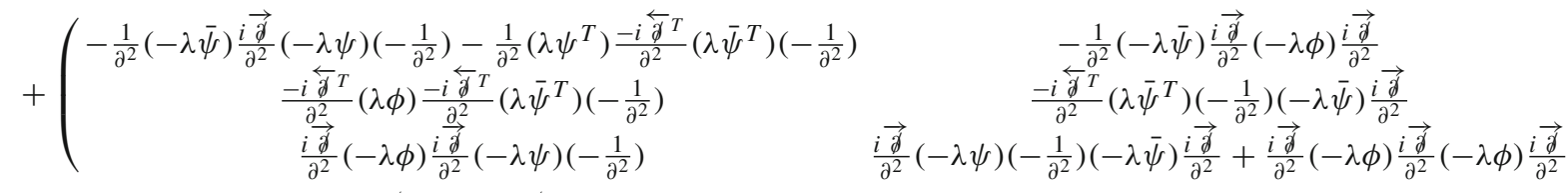

$$
\begin{aligned}
& \left.\begin{array}{c}
-\frac{1}{\partial^{2}}\left(\lambda \psi^{T}\right) \frac{-i \overleftarrow{\not} T}{\partial^{2}}(\lambda \phi) \frac{-i \overleftarrow{\not} T}{\partial^{2}} \\
\frac{-i \overleftarrow{\not} T}{\partial^{2}}\left(\lambda \bar{\psi}^{T}\right)\left(-\frac{1}{\partial^{2}}\right)\left(\lambda \psi^{T}\right) \frac{-i \overleftarrow{\not \partial} T}{\partial^{2}}+\frac{-i \overleftarrow{\not}{ }^{2}}{\partial^{2}}(\lambda \phi) \frac{-i \overleftarrow{\not \partial} T}{\partial^{2}}(\lambda \phi) \frac{-i \overleftarrow{\not} T}{\partial^{2}} \\
\frac{i \vec{\not}}{\partial^{2}}(-\lambda \psi)\left(-\frac{1}{\partial^{2}}\right)\left(\lambda \psi^{T}\right) \frac{-i \overleftarrow{\not} T}{\partial^{2}}
\end{array}\right)+\cdots
\end{aligned}
$$

where $\cdots$ represents the higher order terms in the expansion (4.4). From Eq. (4.7), we should note that there are totally $n$ derivatives $-\frac{1}{\partial^{2}}, \frac{-i \overleftarrow{\not \partial} T}{\partial^{2}}$ or $\frac{i \vec{\not}}{\partial^{2}}$ multiplied together in every element of the $n$th order matrix, and the frst order has no $\lambda$, the second order elements are linear in $\lambda$, then the elements of the $n$th order matrix should be proportional to $\lambda^{n-1} \cdot{ }^{9}$ Furthermore, we should note that $D_{22}^{-1}, D_{33}^{-1} \neq 0$ for the corrections of high order terms, which is different from the ordinary Yukawa theory.

After replacing $\not \partial, \partial^{2}$ by $i \not P,-P^{2}$, we can get the concrete expressions of all propagators from (4.7), as shown in Fig. 3, where $\mathcal{D}_{i j}=D_{i j}^{-1}(\partial \rightarrow P)$. We can also derive the expression of vertex of the hard Lagrangian, as drawn in Fig. 4 following the above conventions.

Just as in the previous seciton, we choose to deform the same momenta and impose the same conditions; then the large $z$ behaviors of elements of $D_{0}^{-1}$ are

$$
\begin{aligned}
-\frac{1}{\partial^{2}} & \sim \frac{1}{\hat{P}^{2}}=\frac{1}{P^{2}+2 P \cdot q} \sim \frac{1}{z} \\
\frac{\not \partial}{\partial^{2}} & \sim \frac{\hat{P}}{\hat{P}^{2}}=\frac{\not P+z q}{P^{2}+2 z q \cdot P} \sim \frac{q}{2 q \cdot P},
\end{aligned}
$$

since the elements of $V$ do not contribute, so the deformed correlation function is only dependent on $D_{0}^{-1}$ in the expansion of $D^{-1}$. When we consider higher order terms in the

\footnotetext{
9 The two facts are consistent with (4.4), since every element of $V$ has a $\lambda$ and derivatives are only contained by $D_{0}^{-1}$.
}

where we have used $q_{1} \cdot q_{2}=0$. From the above results, we can conclude that non-vanishing elements of the second order $D_{0}^{-1} V D_{0}^{-1}$ behave as $1 / z$ when $z \rightarrow \infty$, and elements of higher order terms vanish even faster. So when we consider the large $z$ behavior of deformed correlation functions, we should focus on only one of the propagators, $-i \mathcal{D}_{23}^{-1}$, since only the first order term of the expansion of the propagator may contribute.

Now we begin to calculate the $z$-dependence of deformed amplitudes $\widetilde{B}_{3}(z)$ explicitly. For the cases with threeleg deformation, there are only four cases $\left\langle\mathrm{H}_{1} \mathrm{H}_{2} \mathrm{H}_{3}\right\rangle$, $\left\langle H_{1} \Psi_{2 \alpha} \bar{\Psi}_{3 \beta}\right\rangle,\left\langle\Psi_{1 \alpha} \bar{\Psi}_{2 \beta} \Psi_{3 \gamma}\right\rangle$ and $\left\langle\bar{\Psi}_{1 \alpha} \Psi_{2 \beta} \bar{\Psi}_{3 \gamma}\right\rangle$, and the third one $\left\langle\Psi_{1 \alpha} \bar{\Psi}_{2 \beta} \Psi_{3 \gamma}\right\rangle$ is just the Hermitian conjugate of the fourth one $\left\langle\bar{\Psi}_{1 \alpha} \Psi_{2 \beta} \bar{\Psi}_{3 \gamma}\right\rangle$. The procedure is the same as in the previous section: we will draw the corresponding Feynman diagrams, then write down the expressions, and calculate $\widetilde{B}_{3}(z)$ by LSZ reduction.

For the first case $\left\langle\mathrm{H}_{1} \mathrm{H}_{2} \mathrm{H}_{3}\right\rangle$, its corresponding Feynman diagrams are shown in Fig. 5, where we only draw one digram since the other two diagrams can be got by making permutations of (123). Then the deformed correlation function is

$\hat{G}_{3}(z)=i \lambda\left(-i \mathcal{D}_{12}\right)\left(-i \mathcal{D}_{13}\right)\left(-i \mathcal{D}_{11}\right)+\mathcal{P}(123)$

with $\mathcal{P}(123)$ represent the terms got by permuting the three propagators. ${ }^{10}$ Under the LSZ reduction, the deformed cor-

${ }^{10}$ Note that we are not distinguishing the propagators in position or momentum space, but this does not matter because we will always apply LSZ reduction and only use the propagators in momentum space. 
relation function gives the $z$-dependence of deformed amplitude:

$$
\begin{aligned}
& i \widetilde{B}_{3}(z)=\left(i \lim _{\hat{p}_{1}^{2} \rightarrow 0} \hat{p}_{1}^{2}\right)\left(i \lim _{\hat{p}_{2}^{2} \rightarrow 0} \hat{p}_{2}^{2}\right) \\
&\left(i \lim _{\hat{p}_{3}^{2} \rightarrow 0} \hat{p}_{3}^{2}\right) \hat{G}_{3}(z)+\mathcal{P}(123) \\
&=i \lambda \lim _{\hat{p}_{1}^{2} \rightarrow 0} \lim _{\hat{p}_{2}^{2} \rightarrow 0} \lim _{\hat{p}_{3}^{2} \rightarrow 0} \hat{p}_{1}^{2} \hat{p}_{2}^{2} \hat{p}_{3}^{2} \\
& \times\left[\frac{1}{\hat{P}_{3}^{2}}(\lambda \bar{\psi}) \frac{-\hat{P}_{3}}{\hat{P}_{3}^{2}}+\cdots\right]\left[\frac{1}{\hat{P}_{2}^{2}}+\cdots\right] \\
& \times\left[\frac{1}{\hat{P}_{1}^{2}}\left(\lambda \psi^{T}\right) \frac{-\hat{P}_{1}}{\hat{P}_{1}^{2}}+\cdots\right]+\mathcal{P}(123) \\
&=i \lambda\left[(\lambda \bar{\psi}) \frac{-\hat{P}_{3}}{\hat{P}_{3}^{2}}+\cdots\right][1+\cdots] \\
& \\
&+\left[\left(\lambda \psi{ }^{T}\right) \frac{-\hat{P}_{1}}{\hat{P}_{1}^{2}}+\cdots\right] \\
&+\mathcal{P}(123)=i \lambda^{3} \bar{\psi} \psi^{T} \frac{\hat{P}_{3}}{\hat{P}_{3}^{2}} \frac{\hat{P}_{1}}{\hat{P}_{1}^{2}}+\cdots+\mathcal{P}(123)
\end{aligned}
$$

where we just write the first order terms in the expansion of each propagator. When $z \rightarrow \infty$ (see also (4.9)),

$$
\begin{aligned}
& \frac{\hat{P}_{3}}{\hat{P}_{3}^{2}} \frac{\hat{P}_{1}}{\hat{P}_{1}^{2}}=\frac{\hat{p}_{3} \cdot \hat{p}_{1}}{\hat{p}_{3}^{2} \hat{p}_{1}^{2}}=\frac{\left(\hat{p}_{3}+P_{3}\right) \cdot\left(\hat{p}_{1}+P_{1}\right)}{\left(\hat{p}_{3}+P_{3}\right)^{2}\left(\hat{p}_{1}+P_{1}\right)^{2}} \\
& \quad=\frac{\left(p_{3}+P_{3}\right) \cdot\left(p_{1}+P_{1}\right)+z\left[q_{3} \cdot\left(p_{1}+P_{1}\right)+q_{1} \cdot\left(p_{3}+P_{3}\right)\right]}{\left[\left(p_{3}+P_{3}\right)^{2}+z q_{3} \cdot\left(p_{3}+P_{3}\right)\right]\left[\left(p_{1}+P_{1}\right)^{2}+z q_{1} \cdot\left(p_{1}+P_{1}\right)\right]} \\
& \quad \sim \frac{1}{z}
\end{aligned}
$$

where $P_{1}, P_{3}$ appear for the same reasons as we have explained in the previous section, i.e., with the added soft momenta. The above result shows that the leading term vanish in the limit of $z \rightarrow \infty$, and the next terms will also vanish because they contain more derivatives as showed in (4.7).

For the second case $\left\langle H_{1} \Psi_{2 \alpha} \bar{\Psi}_{3 \beta}\right\rangle$, the corresponding Feynman diagrams are showed in Fig. 5. Since there are five contributing digrams, the expression of $\hat{G}_{3}$ is a little complicated,

$$
\begin{aligned}
\hat{G}_{3}(z)= & {\left[\left(-i \mathcal{D}_{23}^{-1}\right)(i \lambda)\left(-i \mathcal{D}_{23}^{-1}\right)\left(-i \mathcal{D}_{11}^{-1}\right)\right.} \\
& +\left(-i \mathcal{D}_{23}^{-1}\right)(i \lambda)\left(-i \mathcal{D}_{12}^{-1}\right)\left(-i \mathcal{D}_{13}^{-1}\right) \\
& +\left(-i \mathcal{D}_{13}^{-1}\right)(i \lambda)\left(-i \mathcal{D}_{23}^{-1}\right)\left(-i \mathcal{D}_{12}^{-1}\right) \\
& +\left(-i \mathcal{D}_{13}^{-1}\right)(i \lambda)\left(-i \mathcal{D}_{22}^{-1}\right)\left(-i \mathcal{D}_{13}^{-1}\right) \\
& \left.+\left(-i \mathcal{D}_{33}^{-1}\right)(i \lambda)\left(-i \mathcal{D}_{12}^{-1}\right)\left(-i \mathcal{D}_{12}^{-1}\right)\right]
\end{aligned}
$$

After LSZ reduction, we get the $z$-dependence of the 3deformed amplitude,

$$
\begin{aligned}
i \widetilde{B}_{3}(z)= & i \lambda\left(\lim _{\hat{p}_{1}^{2} \rightarrow 0} \hat{p}_{1}^{2}\right)\left(\lim _{\hat{p}_{2}^{2} \rightarrow 0} \hat{p}_{2}\right) \bar{u}_{s_{2}}\left(\hat{p}_{2}\right) \\
& \times\left[\mathcal{D}_{23}^{-1}\left(\hat{P}_{2}\right) \mathcal{D}_{23}^{-1}\left(\hat{P}_{3}\right) \mathcal{D}_{11}^{-1}\left(\hat{P}_{1}\right)\right. \\
& +\mathcal{D}_{23}^{-1}\left(\hat{P}_{2}\right) \mathcal{D}_{12}^{-1}\left(\hat{P}_{3}\right) \mathcal{D}_{13}^{-1}\left(\hat{P}_{1}\right) \\
& +\mathcal{D}_{13}^{-1}\left(\hat{P}_{2}\right) \mathcal{D}_{23}^{-1}\left(\hat{P}_{3}\right) \mathcal{D}_{12}^{-1}\left(\hat{P}_{1}\right) \\
& +\mathcal{D}_{13}^{-1}\left(\hat{P}_{1}\right) \mathcal{D}_{22}^{-1}\left(\hat{P}_{3}\right) \mathcal{D}_{13}^{-1}\left(\hat{P}_{2}\right) \\
& \left.+\mathcal{D}_{33}^{-1}\left(\hat{P}_{2}\right) \mathcal{D}_{12}^{-1}\left(\hat{P}_{3}\right) \mathcal{D}_{12}^{-1}\left(\hat{P}_{1}\right)\right] v_{s_{3}}\left(\hat{p}_{3}\right) \lim _{\hat{p}_{3}^{2} \rightarrow 0} \hat{p}_{3},
\end{aligned}
$$

where $s_{2}, s_{3}$ label the helicities of fermions. In the above formula the leading order is given by the first term with two $\mathcal{D}_{23}^{-1}$ as

$$
\begin{aligned}
& \left(\lim _{\hat{p}_{1}^{2} \rightarrow 0} \hat{p}_{1}^{2}\right)\left(\lim _{\hat{p}_{2}^{2} \rightarrow 0} \hat{p}_{2}\right) \bar{u}_{s_{2}}\left(\hat{p}_{2}\right) \frac{\hat{p}_{2}^{T}}{\hat{p}_{2}^{2}} \frac{\hat{p}_{3}^{T}}{\hat{p}_{3}^{2}} \frac{1}{\hat{p}_{1}^{2}} v_{s_{3}}\left(\hat{p}_{3}\right) \\
& \quad \lim _{\hat{p}_{3}^{2} \rightarrow 0} \hat{p}_{3} \sim \bar{u}_{s_{2}}\left(\hat{p}_{2}\right) v_{s_{3}}\left(\hat{p}_{3}\right),
\end{aligned}
$$

then the large $z$ behavior of $\widetilde{B}_{3}(z)$ depends on the external wavefunction of fermions. The reason why the first term in (4.14) contributes as the leading order is that its three propagators are all in the first order in the expansion of $D^{-1}$ with the least derivatives. For example, in four dimensions, if we make a three-leg deformation like (5.16), the external wavefunctions are also deformed as showed in [21], then the leading order is at least of the order $O\left(z^{0}\right)$. So we conclude that $\widetilde{B}_{3}(z)$ always has non-zero boundary contributions in four dimensions. ${ }^{11}$

For the third case $\left\langle\Psi_{1 \alpha} \bar{\Psi}_{2 \beta} \Psi_{3 \gamma}\right\rangle$, there are three Feynman diagrams contributing as showed in Fig. 6. The deformed correlation function $\hat{G}_{3}$ is

$$
\begin{aligned}
\hat{G}_{3}(z)= & \left(-i \mathcal{D}_{13}^{-1}\right)(i \lambda)\left(-i \mathcal{D}_{22}^{-1}\right)\left(-i \mathcal{D}_{23}^{-1}\right) \\
& +\left(-i \mathcal{D}_{33}^{-1}\right)\left(i \lambda\left(-i \mathcal{D}_{22}^{-1}\right)\left(-i \mathcal{D}_{12}^{-1}\right)\right. \\
& +\left(-i \mathcal{D}_{23}^{-1}\right)(i \lambda)\left(-i \mathcal{D}_{23}^{-1}\right)\left(-i \mathcal{D}_{12}^{-1}\right)
\end{aligned}
$$

After LSZ reduction,

$$
\begin{aligned}
i \widetilde{B}_{3}(z)= & \left(i \lim _{\hat{p}_{1}^{2} \rightarrow 0} \hat{p}_{1}\right) \bar{u}_{s_{1}}\left(\hat{p}_{1}\right)\left(i \lim _{\hat{p}_{3}^{2} \rightarrow 0} \hat{p}_{3}\right) \bar{u}_{s_{3}}\left(\hat{p}_{3}\right) \\
& \times \hat{G}_{3}(z)\left(i \lim _{\hat{p}_{2}^{2} \rightarrow 0} \hat{p}_{2}\right) v_{s_{2}}\left(\hat{p}_{2}\right)
\end{aligned}
$$

\footnotetext{
11 In general dimensions, the deformation of external wavefunctions are complicated, then we do not talk about these here.
} 
Fig. 5 The Feynman diagram (1) is for $\left\langle H_{1} H_{2} H_{3}\right\rangle$ in soft digarms are all for $\left\langle H_{1} \Psi_{2 \alpha} \bar{\Psi}_{3 \beta}\right\rangle$ background, while the last

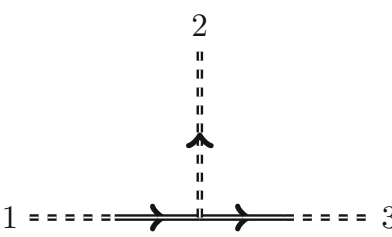

(1)

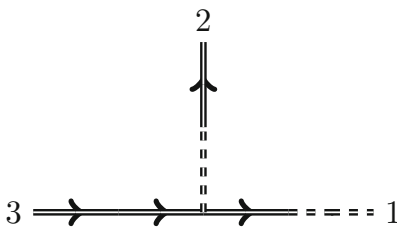

(2.c)

just like in the previous case we only need to focus on the third term and analyze the large $z$ behavior of it, since the term contains the propagator $\mathcal{D}_{12}^{-1}$, which contributes as $O(z)$ after LSZ reduction. So when considering the contributions from the wavefunctions, in some helicity configurations $\widetilde{B}_{3}(z)$ vanishes, but also in some other helicity configurations non-zero boundary contributions appear. As for the last case $\left\langle\bar{\Psi}_{1 \alpha} \Psi_{2 \beta} \bar{\Psi}_{3 \gamma}\right\rangle$, whose Feynman diagrams are shown in Fig. 6 , the discussions are the same as for the third case, since the two are related by complex conjugation.

\section{Yang-Mills theory}

In this section we apply the same method to Yang-Mills theory. In [8], the author used the background field method by splitting the YM field into a hard field and a soft field, but only wrote down quadratic terms of the hard field since one only considered the significance of propagators of hard field. However, because we consider the on-shell recursion relations of the three-legs deformed case, we need to calculate the interaction part of the hard field. As before, we consider the Lagrangian of the pure Yang-Mills theory,

$\mathcal{L}=-\frac{1}{4} F_{\mu \nu}^{c} F^{\mu \nu c}$

If we split the Yang-Mills field $A_{\mu}$ into $A_{\mu} \rightarrow a_{\mu}+A_{\mu}$, where $a_{\mu}$ is the soft field and $A_{\mu}$ is the hard field, the field strength becomes

$F_{\mu \nu}^{c} \rightarrow \bar{F}_{\mu \nu}^{c}+\left(\overline{\mathcal{D}}_{\mu} A_{\nu}\right)^{c}-\left(\overline{\mathcal{D}}_{\nu} A_{\mu}\right)^{c}+g f^{a b c} A_{\mu}^{a} A_{\nu}^{b}$,

where $\bar{F}_{\mu \nu}^{c}$ is the field strength for the soft field and $\overline{\mathcal{D}}_{\mu}^{a b}=$ $\partial_{\mu} \delta^{a b}-g f^{c a b} a_{\mu}^{c}$ is the background covariant derivative. Substituting it into the Lagrangian, we obtain

$\mathcal{L}(a, A)=-\frac{1}{2}\left(\overline{\mathcal{D}}_{\mu} A_{\nu}\right)^{a}\left(\overline{\mathcal{D}}^{\mu} A^{v}\right)^{a}$
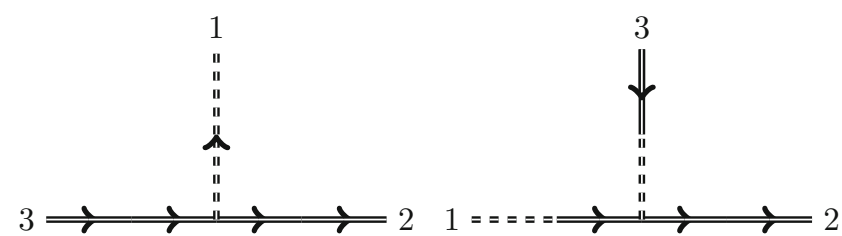

(2.a)

$(2 . b)$
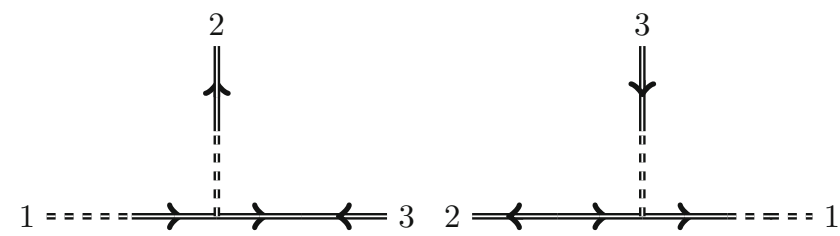

$(2 . d)$

(2.e)

$+\frac{1}{2}\left(\overline{\mathcal{D}}_{\mu} A_{\nu}\right)^{a}\left(\overline{\mathcal{D}}^{\nu} A^{\mu}\right)^{a}$

$-\frac{1}{2} g f^{a b c} \bar{F}^{c \mu v} A_{\mu}^{a} A_{v}^{b}$

$-g f^{a b c} A^{a \mu} A^{b v}\left(\overline{\mathcal{D}}_{\mu} A_{\nu}\right)^{c}$

$-\frac{1}{4} g^{2} f^{a b e} f^{c d e} A^{a \mu} A^{b v} A_{\mu}^{c} A_{v}^{d}$,

where the terms without $A$ or linear in $A$ have been dropped. Then we consider adding the gauge-fixing term, ${ }^{12}$

$$
\begin{aligned}
\mathcal{L}_{g f} & =-\frac{1}{2} \xi^{-1}\left(\overline{\mathcal{D}}^{\mu} A_{\mu}\right)^{a}\left(\overline{\mathcal{D}}^{v} A_{\nu}\right)^{a} \\
& =-\frac{1}{2}\left(\overline{\mathcal{D}}^{\mu} A^{v}\right)^{c}\left(\overline{\mathcal{D}}_{\nu} A_{\mu}\right)^{c}-\frac{1}{2} g f^{a b c} \bar{F}^{c \mu \nu} A_{\mu}^{a} A_{v}^{b}
\end{aligned}
$$

with $\xi=1$. So we get

$$
\begin{aligned}
\mathcal{L}(a, A)+\mathcal{L}_{g f}= & -\frac{1}{2}\left(\overline{\mathcal{D}}_{\mu} A_{\nu}\right)^{a}\left(\overline{\mathcal{D}}^{\mu} A^{\nu}\right)^{a} \\
& -g f^{a b c} \bar{F}^{c \mu v} A_{\mu}^{a} A_{\nu}^{b} \\
& -g f^{a b c} A^{a \mu} A^{b v}\left(\overline{\mathcal{D}}_{\mu} A_{\nu}\right)^{c} \\
& -\frac{1}{4} g^{2} f^{a b e} f^{c d e} A^{a \mu} A^{b v} A_{\mu}^{c} A_{\nu}^{d},
\end{aligned}
$$

then we can get the expression of the propagator from the first line and vertices from the second line.

First, to write down the expression of propagator, we need to consider the first line of (5.5) and after using integration by parts we get

$$
\begin{aligned}
M_{\mu \nu}^{a b}= & \left(\overline{\mathcal{D}}^{\rho} \overline{\mathcal{D}}_{\rho}\right)^{a b} g_{\mu \nu}-2 g f^{a b c} \bar{F}_{\mu \nu}^{c} \\
= & g_{\mu \nu} \delta^{a b} \partial^{2}+g f^{a b c} g_{\mu \nu}\left(\partial^{\rho} a_{\rho}^{c}\right) \\
& +2 g f^{a b c} g_{\mu \nu} a_{\rho}^{c} \partial^{\rho}-2 g f^{a b c} \bar{F}_{\mu \nu}^{c} \\
& +g^{2} f^{a d c} f^{c e b} a_{\rho}^{d} a^{\rho e} g_{\mu \nu}
\end{aligned}
$$

$\overline{12}$ We do not consider the ghost term for our considerations are limited in the tree diagrams. 
Fig. 6 The three Feynman diagrams in the first line are for $\left\langle\Psi_{1 \alpha} \bar{\Psi}_{2 \beta} \Psi_{3 \gamma}\right\rangle$, the three Feynman diagrams in the second line are for $\left\langle\bar{\Psi}_{1 \alpha} \Psi_{2 \beta} \bar{\Psi}_{3 \gamma}\right\rangle$

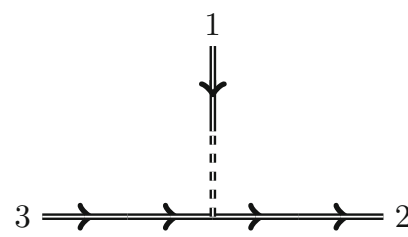

(3.a)

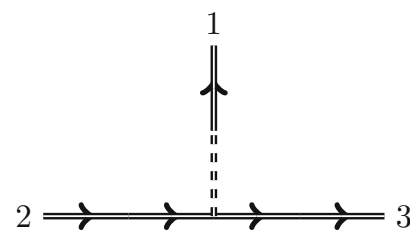

(4.a)

$$
=g_{\mu \nu} \delta^{a b} \partial^{2}-V_{\mu \nu}^{a b}
$$

where $V_{\mu \nu}^{a b}=-g f^{a b c} g_{\mu \nu}\left(\partial^{\rho} a_{\rho}^{c}\right)-2 g f^{a b c} g_{\mu \nu} a_{\rho}^{c} \partial^{\rho}+$ $2 g f^{a b c} \bar{F}_{\mu \nu}^{c}-g^{2} f^{a d c} f^{c e b} a_{\rho}^{d} a^{\rho e} g_{\mu \nu}$. Then we take the inverse of $M_{\mu \nu}^{a b}$ formlly as

$$
\begin{aligned}
\left(M_{\mu \nu}^{a b}\right)^{-1}= & \left(g_{\mu \nu} \delta^{a b} \partial^{2}-V_{\mu \nu}^{a b}\right)^{-1} \\
= & \left\{\partial^{2}\left[g_{\mu \nu} \delta^{a b}-\left(\partial^{2}\right)^{-1} V_{\mu \nu}^{a b}\right]\right\}^{-1} \\
= & {\left[g^{\mu \nu} \delta^{a b}-\left(\partial^{2}\right)^{-1} V^{a b, \mu \nu}\right.} \\
& \left.+\left(\partial^{2}\right)^{-1} V^{a c, \mu \rho}\left(\partial^{2}\right)^{-1} V^{c b, \rho \nu}+\cdots\right]\left(\partial^{2}\right)^{-1}
\end{aligned}
$$

the momentum is got by replacing $\partial^{2}, \partial_{\mu}$ by $-p^{2}, i p_{\mu}$. Second, from (5.5), we know there are two kinds of cubic vertices and one quartic vertex; we need to write the explicit expressions of these vertices one by one. The first cubic vertex contains three hard momenta only and is given by

$$
\begin{aligned}
i V_{\mu \nu \rho}^{a b c}(p, q, r)= & g f^{a b c}\left[(q-r)_{\mu} g_{\nu \rho}\right. \\
& \left.+(r-p)_{\nu} g_{\rho \mu}+(p-q)_{\rho} g_{\mu \nu}\right] .
\end{aligned}
$$

The second cubic vertex connects three hard fields and one soft field,

$$
\begin{aligned}
i V_{\mu \nu \rho}^{a b c}(a)= & -i g^{2}\left[f^{a b d} f^{d e c}\left(a_{\mu}^{e} g_{\nu \rho}-a_{\nu}^{e} g_{\mu \rho}\right)\right. \\
& +f^{a c d} f^{d e b}\left(a_{\mu}^{e} g_{\nu \rho}-a_{\rho}^{e} g_{\mu \nu}\right) \\
& \left.+f^{b c d} f^{d e a}\left(a_{\nu}^{e} g_{\mu \rho}-a_{\rho}^{e} g_{\mu \nu}\right)\right]
\end{aligned}
$$

Although we will not use the quartic vertex $i V_{\mu \nu \rho \sigma}^{a b c d}$ of the hard fields in this paper, when four or more momenta are deformed, the quartic vertex should be included to analyze the boundary behavior, so we give its expression,

$i V_{\mu \nu \rho \sigma}^{a b c d}=-i g^{2}\left[f^{a b e} f^{c d e}\left(g_{\mu \rho} g_{\nu \sigma}-g_{\mu \sigma} g_{\nu \rho}\right)\right.$

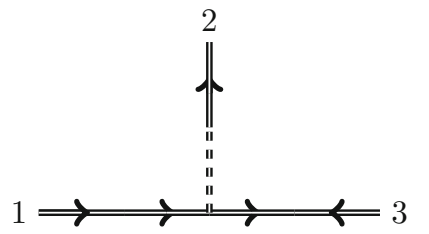

(3.b)

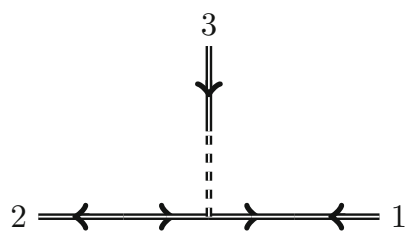

(3.c)
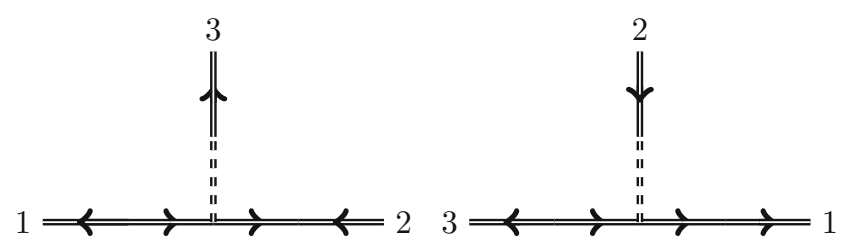

(4.b)

(4.c)
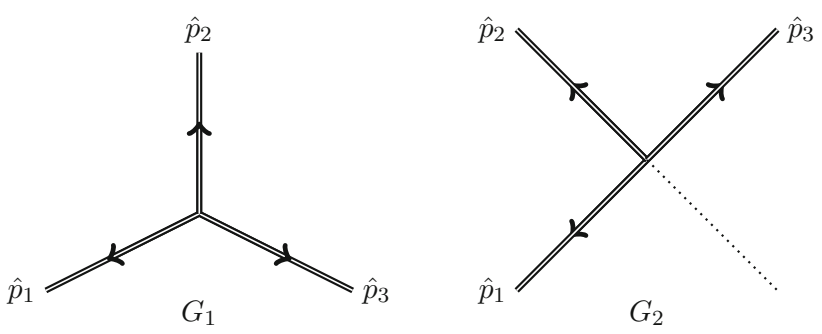

Fig. 7 The Feynman diagrams with three hard lines for deformed correlation function in Yang-Mills theory

$$
\begin{aligned}
& +f^{a d e} f^{b c e}\left(g_{\mu \nu} g_{\sigma \rho}-g_{\mu \rho} g_{\sigma \nu}\right) \\
& \left.+f^{a c e} f^{d b e}\left(g_{\mu \sigma} g_{\rho \nu}-g_{\mu \nu} g_{\rho \sigma}\right)\right],
\end{aligned}
$$

for completeness.

Having obtained the Feynman rules, we begin to calculate the deformed correlation function of an amplitude. Since we only deform three external momenta, we need only consider these diagrams with three hard particles. So there are only two diagrams contributing as shown in Fig. 7, the first is the one given by the first kind of cubic vertex, denoted by $G_{1}$, the second is the one given by the second kind of cubic vertex denoted by $G_{2}$.

The expressions of the two diagrams are given as

$$
\begin{aligned}
\hat{G}_{3}^{d e f, \alpha \beta \gamma}(z)= & i V_{\mu \nu \rho}^{a b c}\left[-i\left(M^{-1}\right)^{a d, \mu \alpha}\left(\hat{p}_{1}\right)\right] \\
& \times\left[-i\left(M^{-1}\right)^{b e, \nu \beta}\left(\hat{p}_{2}\right)\right]\left[-i\left(M^{-1}\right)^{c f, \rho \gamma}\left(\hat{p}_{3}\right)\right] \\
& +i V_{\mu \nu \rho}^{a b c}(a)\left[-i\left(M^{-1}\right)^{a d, \mu \alpha}\left(\hat{p}_{1}\right)\right] \\
& \times\left[-i\left(M^{-1}\right)^{b e, \nu \beta}\left(\hat{p}_{2}\right)\right]\left[-i\left(M^{-1}\right)^{c f, \rho \gamma}\left(\hat{p}_{3}\right)\right] .
\end{aligned}
$$


Then we do the LSZ reduction and get the boundary contribution of the three-leg deformed amplitude,

$$
\begin{aligned}
\widetilde{B}_{3}(z)= & \left(i \lim _{\hat{p}_{1}^{2} \rightarrow 0} \hat{p}_{1}^{2}\right)\left(i \lim _{\hat{p}_{2}^{2} \rightarrow 0} \hat{p}_{2}^{2}\right)\left(i \lim _{\hat{p}_{3}^{2} \rightarrow 0} \hat{p}_{3}^{2}\right) \\
& \times \hat{G}_{3}^{d e f, \alpha \beta \gamma}(z) \epsilon_{s_{1}}^{\alpha}\left(\hat{p}_{1}\right) \epsilon_{s_{2}}^{\beta}\left(\hat{p}_{2}\right) \epsilon_{S_{3}}^{\gamma}\left(\hat{p}_{3}\right),
\end{aligned}
$$

where $s_{i}$ represents the helicity of the $i$ th particle. Since there are two diagrams contributing, we discuss them one by one. The first contribution comes from the first line of (5.10),

$$
\begin{aligned}
\widetilde{B}_{3}^{(1)}(z)= & \left(\lim _{\hat{p}_{1}^{2} \rightarrow 0} \hat{p}_{1}^{2}\right)\left(\lim _{\hat{p}_{2}^{2} \rightarrow 0} \hat{p}_{2}^{2}\right)\left(\lim _{\hat{p}_{3}^{2} \rightarrow 0} \hat{p}_{3}^{2}\right) V_{\mu \nu \rho}^{a b c} \\
& \times\left[\left(M^{-1}\right)^{a d, \mu \alpha}\left(\hat{p}_{1}\right)\right]\left[\left(M^{-1}\right)^{b e, \nu \beta}\left(\hat{p}_{2}\right)\right] \\
& \times\left[\left(M^{-1}\right)^{c f, \rho \gamma}\left(\hat{p}_{3}\right)\right] \epsilon_{s_{1}}^{\alpha}\left(\hat{p}_{1}\right) \epsilon_{s_{2}}^{\beta}\left(\hat{p}_{2}\right) \epsilon_{s_{3}}^{\gamma}\left(\hat{p}_{3}\right) \\
= & g f^{a b c}\left[\left(\hat{p}_{2}-\hat{p}_{3}\right)_{\mu} g_{v \rho}+\left(\hat{p}_{3}-\hat{p}_{1}\right)_{\nu} g_{\rho \mu}\right. \\
& \left.+\left(\hat{p}_{1}-\hat{p}_{2}\right)_{\rho} g_{\mu \nu}\right] \epsilon_{s_{1}}^{\alpha}\left(\hat{p}_{1}\right) \epsilon_{s_{2}}^{\beta}\left(\hat{p}_{2}\right) \epsilon_{s_{3}}^{\gamma}\left(\hat{p}_{3}\right) \\
& \times\left[g^{\mu \alpha} \delta^{a d}-\left(\hat{p}_{1}^{2}\right)^{-1} V^{a d, \mu \alpha}+\cdots\right] \\
& \times\left[g^{\nu \beta} \delta^{b e}-\left(\hat{p}_{2}^{2}\right)^{-1} V^{b e, \nu \beta}+\cdots\right] \\
& \times\left[g^{\rho \gamma} \delta^{c f}-\left(\hat{p}_{1}^{2}\right)^{-1} V^{c f, \rho \gamma}+\cdots\right]
\end{aligned}
$$

If we expand the above equation and look at the first order term, we obtain

$$
f^{\text {def }}\left[\left(\hat{p}_{2}-\hat{p}_{3}\right) \cdot \epsilon_{s_{1}}\left(\hat{p}_{1}\right)\right]\left[\epsilon_{s_{2}}\left(\hat{p}_{2}\right) \cdot \epsilon_{s_{3}}\left(\hat{p}_{3}\right)\right]+\mathcal{P}(123) .
$$

For the higher order terms, although $V^{a d, \mu \alpha}$ may contribute in the order $z,\left(\hat{p}_{2}^{2}\right)^{-1}$ is also $z^{-1}$, so higher order terms contribute the same order as the first ones. So the large $z$ behavior of $\hat{A}_{3}^{(1)}(z)$ is more complicated, which depends on the $z$ behaviors of polarization vectors as well as 3-deformed momenta. The contribution from the second diagram is

$$
\begin{aligned}
\widetilde{B}_{3}^{(2)}(z)= & \left(\lim _{\hat{p}_{1}^{2} \rightarrow 0} \hat{p}_{1}^{2}\right)\left(\lim _{\hat{p}_{2}^{2} \rightarrow 0} \hat{p}_{2}^{2}\right)\left(\lim _{\hat{p}_{3}^{2} \rightarrow 0} \hat{p}_{3}^{2}\right) V_{\mu \nu \rho}^{a b c}(a) \\
& \times\left[\left(M^{-1}\right)^{a d, \mu \alpha}\left(\hat{p}_{1}\right)\right]\left[\left(M^{-1}\right)^{b e, \nu \beta}\left(\hat{p}_{2}\right)\right] \\
& \times\left[\left(M^{-1}\right)^{c f, \rho \gamma}\left(\hat{p}_{3}\right)\right] \epsilon_{s_{1}}^{\alpha}\left(\hat{p}_{1}\right) \epsilon_{s_{2}}^{\beta}\left(\hat{p}_{2}\right) \epsilon_{s_{3}}^{\gamma}\left(\hat{p}_{3}\right) \\
= & -g^{2}\left[f^{a b c} f^{c e d}\left(a_{\mu}^{e} g_{\nu \rho}-a_{\nu}^{e} g_{\mu \rho}\right)\right. \\
& +f^{a d c} f^{c e b}\left(a_{\mu}^{e} g_{\nu \rho}-a_{\rho}^{e} g_{\mu \nu}\right) \\
& \left.+f^{b d c} f^{c e a}\left(a_{\nu}^{e} g_{\mu \rho}-a_{\rho}^{e} g_{\mu \nu}\right) \epsilon_{s_{1}}^{\alpha}\left(\hat{p}_{1}\right) \epsilon_{s_{2}}^{\beta}\left(\hat{p}_{2}\right) \epsilon_{s_{3}}^{\gamma}\left(\hat{p}_{3}\right)\right] \\
& \times\left[g^{\mu \alpha} \delta^{a d}-\left(\hat{p}_{1}^{2}\right)^{-1} V^{a d, \mu \alpha}+\cdots\right]
\end{aligned}
$$

$$
\begin{aligned}
& \times\left[g^{\nu \beta} \delta^{b e}-\left(\hat{p}_{2}^{2}\right)^{-1} V^{b e, \nu \beta}+\cdots\right] \\
& \times\left[g^{\rho \gamma} \delta^{c f}-\left(\hat{p}_{1}^{2}\right)^{-1} V^{c f, \rho \gamma}+\cdots\right],
\end{aligned}
$$

where terms of the first order are

$$
\begin{gathered}
f^{\text {def }} f^{\text {fed }}\left\{\left[a \cdot \epsilon_{s_{1}}\left(\hat{p}_{1}\right)\right]\left[\epsilon_{s_{2}}\left(\hat{p}_{2}\right) \cdot \epsilon_{s_{3}}\left(\hat{p}_{3}\right)\right]\right. \\
\left.-\left[a \cdot \epsilon_{s_{2}}\left(\hat{p}_{2}\right)\right]\left[\epsilon_{s_{1}}\left(\hat{p}_{1}\right) \cdot \epsilon_{s_{3}}\left(\hat{p}_{3}\right)\right]\right\},
\end{gathered}
$$

and higher order terms behave similar to the terms mentioned before. Combining the two contributions, since the first contribution depends on deformed momenta and always has larger order of $z$ than the second one, when we analyze the large $z$ behavior of amplitude, we need only consider the first diagram and ignore the second one.

After calculating the boundary terms, to specify the explicit boundary behavior of amplitude, we should know the $z$-dependences of the polarization vectors. Since the $z$ dependence of polarization vectors in the three-leg deformed (or even more deformed) case are not so easily determined, we take an amplitude in four dimensions as an example. The three-leg deformation is chosen as ${ }^{13}$

$$
\begin{aligned}
& |\hat{1}\rangle=|1\rangle-z|2\rangle-z|3\rangle, \quad \mid \hat{1}]=\mid 1], \\
& \mid \hat{2}]=\mid 2]+z \mid 1], \quad|\hat{2}\rangle=|2\rangle, \\
& \mid \hat{3}]=\mid 3]+z \mid 1], \quad|\hat{3}\rangle=|3\rangle .
\end{aligned}
$$

Next we write down the corresponding polarization vectors,

$$
\begin{aligned}
& \epsilon_{1}^{-}(z)=-\frac{\left\langle\hat{1}\left|\gamma^{\mu}\right| q_{1}\right]}{\sqrt{2}\left[q_{1} \hat{1}\right]} \epsilon_{1}^{+}(z)=-\frac{\left\langle q_{1}\left|\gamma^{\mu}\right| \hat{1}\right]}{\sqrt{2}\left\langle q_{1} \hat{1}\right\rangle}, \\
& \epsilon_{2}^{-}(z)=-\frac{\left\langle\hat{2}\left|\gamma^{\mu}\right| q_{2}\right]}{\sqrt{2}\left[q_{2} \hat{2}\right]} \quad \epsilon_{2}^{+}(z)=-\frac{\left\langle q_{2}\left|\gamma^{\mu}\right| \hat{2}\right]}{\sqrt{2}\left\langle q_{2} \hat{2}\right\rangle}, \\
& \epsilon_{3}^{-}(z)=-\frac{\left\langle\hat{3}\left|\gamma^{\mu}\right| q_{3}\right]}{\sqrt{2}\left[q_{3} \hat{3}\right]} \quad \epsilon_{3}^{+}(z)=-\frac{\left\langle q_{3}\left|\gamma^{\mu}\right| \hat{3}\right]}{\sqrt{2}\left\langle q_{3} \hat{3}\right\rangle} .
\end{aligned}
$$

From the above form of the polarization vectors, we can know that the polarization vectors $\epsilon_{1}^{-}(z), \epsilon_{2}^{+}(z), \epsilon_{3}^{+}(z)$ are of order $\mathcal{O}(z)$, while the polarization vectors $\epsilon_{1}^{+}(z), \epsilon_{2}^{-}(z), \epsilon_{3}^{-}(z)$ are of order $O\left(z^{-1}\right)$. There are in total 8 helicity configurations for the three particles; since the analyses for all cases are the same, we focus on one case, $\left(1^{+}, 2^{-}, 3^{-}\right)$, to illustrate our method. When $z \rightarrow \infty$, the leading contributions for the amplitude of the helicity configuration are the leading terms in the first diagram,

$$
\begin{aligned}
& {\left[\left(\hat{p}_{2}-\hat{p}_{3}\right) \cdot \epsilon_{1}^{+}(z)\right]\left[\epsilon_{2}^{-}(z) \cdot \epsilon_{3}^{-}(z)\right]} \\
& \quad+\left[\left(\hat{p}_{3}-\hat{p}_{1}\right) \cdot \epsilon_{2}^{-}(z)\right]\left[\epsilon_{3}^{-}(z) \cdot \epsilon_{1}^{+}(z)\right]
\end{aligned}
$$

13 We can easily check that they satisfy the previous mentioned conditions. Here the conventions follow [21]. 


$$
+\left[\left(\hat{p}_{1}-\hat{p}_{2}\right) \cdot \epsilon_{3}^{-}(z)\right]\left[\epsilon_{1}^{+}(z) \cdot \epsilon_{2}^{-}(z)\right] .
$$

If we choose $q_{2}=q_{3}$, then the first term in the above formula vanish for $\left[\epsilon_{2}^{-}(z) \cdot \epsilon_{3}^{-}(z)\right]=0$, and after calculations we can see that $\left[\epsilon_{3}^{-}(z) \cdot \epsilon_{1}^{+}(z)\right] \sim 1 / z^{2},\left[\epsilon_{1}^{+}(z) \cdot \epsilon_{2}^{-}(z)\right] \sim 1 / z^{2}$ and $\left[\left(\hat{p}_{3}-\hat{p}_{1}\right) \cdot \epsilon_{2}^{-}(z)\right] \sim z,\left[\left(\hat{p}_{1}-\hat{p}_{2}\right) \cdot \epsilon_{3}^{-}(z)\right] \sim z$. So when $z \rightarrow \infty$, the boundary terms vanish for a gluon amplitude with $\left(1^{+}, 2^{-}, 3^{-}\right)$for 3 -deformed particles. However, we should note that, for other helicity configurations, the boundary terms do not always vanish, which can be inferred from the $z$ dependences of $\epsilon_{1}^{-}(z), \epsilon_{2}^{+}(z), \epsilon_{3}^{+}(z)$.

\section{Conclusion}

The vanishing of the boundary contribution $B_{n}$ of a deformed amplitude $\hat{A}_{n}$ is vital for the existence of on-shell recursion relations; thus much literature has paid special attention to analyzing how a deformed amplitude $\hat{A}_{n}$ behaves when $z$ approaches infinity and to determining when these recursion relations are applicable. However, in general the boundary contribution is unavoidable in many theories, so the understanding of the boundary becomes an interesting problem.

In this paper, we try to calculate the $z$-denpendence of a deformed amplitude by using the background field method in the case of multiple legs being deformed. The method relies on the key idea proposed in $[1,8]$ that we can view the particles with momenta being deformed as hard particles while we can view the others as soft particles when $z \rightarrow \infty$, and the deformed amplitude is the description of hard particles scattering in the background of soft particles. To apply the interpretation to practical calculations, we need another tool, LSZ reduction, to relate the deformed amplitude with its corresponding deformed correlation function in [2], where hard particles correspond to fields with deformed momenta. Once the correspondence is established, the computation of the $z$ dependence of the deformed amplitude is transformed into a simple computation of the sum of several Feynman diagrams, which exactly depict hard particles scattering in the soft background. The key point is to write down the correct Feynman rules for the hard Lagrangian, draw the corresponding Feynman diagrams and calculate the expressions of them. The whole procedure is the same as what we learn from any standard QFT textbook, so the method is very simple. Although in this paper we are limited to the case with only three external legs being deformed, it can also be generalized to situations where more legs are deformed.

After having given the general discussions of how to combine the background field method with on-shell recursion relations, we presented three examples to illustrate our method, ranging from the simplest one, real scalar theory, to more complicated Yukawa theory and pure YM theory. There are some noteworthy features of these examples. First, the Feynman diagrams in the background field method are actually the combination of all diagrams which depict the real scattering process without background, so it is possible to get the explicit expressions of the $z$-dependent part of deformed amplitude. When we expand the propagator in a soft background, we can find these diagrams to depict real scattering process again. Secondly, the large $z$ contributions are dominated by the leading terms of the expansion of the propagators in soft background, especially those with less derivatives, because these derivatives will produce some $\hat{p}_{i}^{2} \sim z$ in denominators. Thirdly, the external wave functions are also important and actually determine if the on-shell recursion relations exist in some critical cases, just as shown by those examples in Yukawa theory and YM theory.

Acknowledgements We thank Bo Feng for giving us the idea and helpful discussions. We would also like to thank Qingjun Jing, Rijun Huang, Junjie Rao for helpful discussions. This work is support by Qiu-Shi Funding and the National Natural Science Foundation of China (NSFC) with Grant No. 11935013, No. 11575156.

Data Availability Statement This manuscript has no associated data or the data will not be deposited. [Authors' comment: This paper is to develop the theoretical method of calculating the boundary contributions of multiple-leg deformed amplitudes, and we provide three examples to illustrate the method, so it doesn't need experiment data.]

Open Access This article is licensed under a Creative Commons Attribution 4.0 International License, which permits use, sharing, adaptation, distribution and reproduction in any medium or format, as long as you give appropriate credit to the original author(s) and the source, provide a link to the Creative Commons licence, and indicate if changes were made. The images or other third party material in this article are included in the article's Creative Commons licence, unless indicated otherwise in a credit line to the material. If material is not included in the article's Creative Commons licence and your intended use is not permitted by statutory regulation or exceeds the permitted use, you will need to obtain permission directly from the copyright holder. To view a copy of this licence, visit http://creativecomm ons.org/licenses/by/4.0/.

Funded by SCOAP ${ }^{3}$.

\section{References}

1. N. Arkani-Hamed, J. Kaplan, On tree amplitudes in gauge theory and gravity. JHEP 04, 076 (2008). https://doi.org/10.1088/ 1126-6708/2008/04/076. arXiv:0801.2385

2. Q. Jin, B. Feng, Boundary operators of BCFW recursion relation. JHEP. 04, 123 (2016). https://doi.org/10.1007/JHEP04(2016)123. arXiv: 1507.00463

3. R. Britto, F. Cachazo, B. Feng, New recursion relations for tree amplitudes of gluons. Nucl. Phys. B. 715, 499 (2005). https://doi. org/10.1016/j.nuclphysb.2005.02.030. arXiv:hep-th/0412308

4. R. Britto, F. Cachazo, B. Feng, E. Witten, Direct proof of treelevel recursion relation in Yang-Mills theory. Phys. Rev. Lett. 94, 181602 (2005). https://doi.org/10.1103/PhysRevLett.94.181602. arXiv:hep-th/0501052

5. K. Risager, A direct proof of the CSW rules. JHEP. 12, 003 (2005). https://doi.org/10.1088/1126-6708/2005/12/003. arXiv:hep-th/0508206 
6. T. Cohen, H. Elvang, M. Kiermaier, On-shell constructibility of tree amplitudes in general field theories. JHEP 04, 053 (2011). https:// doi.org/10.1007/JHEP04(2011)053. arXiv:1010.0257

7. C. Cheung, C.-H. Shen, J. Trnka, Simple recursion relations for general field theories. JHEP 06, 118 (2015). https://doi.org/10. 1007/JHEP06(2015)118. arXiv:1502.05057

8. C. Cheung, On-shell recursion relations for generic theories. JHEP 03, 098 (2010). https://doi.org/10.1007/JHEP03(2010)098. arXiv:0808.0504

9. P. Benincasa, F. Cachazo, Consistency conditions on the S-matrix of massless particles. arXiv:0705.4305

10. R.H. Boels, No triangles on the moduli space of maximally supersymmetric gauge theory. JHEP 05, 046 (2010). https://doi.org/10. 1007/JHEP05(2010)046. arXiv:1003.2989

11. B. Feng, J. Wang, Y. Wang, Z. Zhang, BCFW recursion relation with nonzero boundary contribution. JHEP 01, 019 (2010). https:// doi.org/10.1007/JHEP01(2010)019. arXiv:0911.0301

12. B. Feng, C.-Y. Liu, A note on the boundary contribution with bad deformation in gauge theory. JHEP 07, 093 (2010). https://doi.org/ 10.1007/JHEP07(2010)093. arXiv:1004.1282

13. B. Feng, Z. Zhang, Boundary contributions using fermion pair deformation. JHEP 12, 057 (2011). https://doi.org/10.1007/ JHEP12(2011)057. arXiv:1109.1887

14. K. Zhou, C. Qiao, General tree-level amplitudes by factorization limits. Eur. Phys. J. C. 75, 163 (2015). https://doi.org/10.1140/ epjc/s10052-015-3391-z. arXiv:1410.5042
15. P. Benincasa, E. Conde, On the tree-level structure of scattering amplitudes of massless particles. JHEP 11, 074 (2011). https://doi. org/10.1007/JHEP11(2011)074. arXiv:1106.0166

16. P. Benincasa, E. Conde, Exploring the S-matrix of massless particles. Phys. Rev. D. 86, 025007 (2012). https://doi.org/10.1103/ PhysRevD.86.025007. arXiv: 1108.3078

17. B. Feng, Y. Jia, H. Luo, M. Luo, Roots of amplitudes. arXiv: 1111.1547

18. B. Feng, K. Zhou, C. Qiao, J. Rao, Determination of boundary contributions in recursion relation. JHEP 03, 023 (2015). https:// doi.org/10.1007/JHEP03(2015)023. arXiv:1411.0452

19. B. Feng, J. Rao, K. Zhou, On multi-step BCFW recursion relations. JHEP 07, 058 (2015). https://doi.org/10.1007/JHEP07(2015)058. arXiv: 1504.06306

20. Q. Jin, B. Feng, Recursion relation for boundary contribution. JHEP 06, 018 (2015). https://doi.org/10.1007/JHEP06(2015)018. arXiv: 1412.8170

21. H. Elvang, Y.-t. Huang, Scattering amplitudes. arXiv:1308.1697 